\title{
Aneuploidy influences the gene expression profiles in Saccharomyces pastorianus group I and II strains during fermentation.
}

Roberto de la Cerda ${ }^{1}$, Karsten Hokamp ${ }^{2}$, Fiona Roche ${ }^{2}$, Georgia Thompson ${ }^{1}$, Soukaina

Timouma $^{3}$, Daniela Delneri ${ }^{3}$ and Ursula Bond $*^{1}$

${ }^{1}$ Moyne Institute, School of Genetics and Microbiology, Trinity College Dublin, College

Green, Dublin 2 Ireland.

${ }^{2}$ Smurfit Institute of Genetics, School of Genetics and Microbiology, Trinity College Dublin, College Green, Dublin 2 Ireland.

${ }^{3}$ Manchester Institute of Biotechnology, Faculty of Biology, Medicine and Health, University of Manchester, 131 Princess Street, Manchester M1 7DN, UK

*Corresponding author: ubond@tcd.ie 


\section{Abstract}

2 The lager yeasts, Saccharomyces pastorianus, are hybrids of Saccharomyces cerevisiae and

3 Saccharomyces eubayanus and are divided into two broad groups, Group I and II. The two

4 groups evolved from at least one common hybridisation event but have subsequently

5 diverged with Group I strains losing many S. cerevisiae chromosomes while the Group II

6 strains retain both sub-genomes. The complex genomes, containing orthologous alleles from

7 the parental chromosomes, pose interesting questions regarding gene regulation and its

8 impact on the fermentation properties of the strains. Superimposed on the presence of

9 orthologous alleles are complexities of gene dosage due to the aneuploid nature of the

10 genomes. We examined the contribution of the S. cerevisiae and S. eubayanus alleles to the

11 gene expression patterns of Group I and II strains during fermentation. We show that the

12 relative expression of $S$. cerevisiae and S. eubayanus orthologues is positively correlated with

13 gene copy number. Despite the reduced $S$. cerevisiae content in the Group I strain, $S$.

14 cerevisiae orthologues contribute to biochemical pathways upregulated during fermentation

15 which may explain the retention of specific chromosomes in the strain. Conversely, $S$.

16 eubayanus genes are significantly overrepresented in the upregulated gene pool in the Group

17 II strain. Comparison of the transcription profiles of Group I and II strains during

18 fermentation identified both common and unique gene expression patterns, with gene copy

19 number being a dominant contributory factor. Thus, the aneuploid genomes create complex

20 patterns of gene expression during fermentation with gene dosage playing a crucial role both

21 within and between strains. 


\section{$24 \quad$ Introduction}

25 The yeasts used in lager beer production have long been recognised as being unique and

26 distinct from those used for making ales (1). In 1870, in recognition of their unique

27 physiological qualities, this group of yeast were given a taxonomical classification of

28 Saccharomyces pastorianus by Max Reess (2). After more than a century of genetic studies,

29 we now know that S. pastorianus strains are natural hybrids of Saccharomyces cerevisiae and

30 Saccharomyces eubayanus (3, 4). While S. cerevisiae has long been associated with beer

31 production, especially for ales, S. eubayanus was only discovered in Patagonia, South

32 America, in 2011 (5). While Patagonia is considered to be the primary radiation source of $S$.

33 eubayanus, natural isolates have also been found in North America, China, Tibet and New

34 Zealand but to date, no isolates have been identified in Europe (6-9) with only limited

35 metagenomic evidence for the presence of this species in Italian Alps (10). The hybridisation

36 events that generated the current strains of $S$. pastorianus are estimated to have occurred

37 some 500-600 years ago following the introduction of S. eubayanus into Central Europe from

38 China or Tibet, most likely along trade routes such as the Silk Road. An inherited property of

39 cryotolerance from the $S$. eubayanus genome allows $S$. pastorianus strains to ferment at

40 temperatures as low as $7-13^{\circ} \mathrm{C}(11)$. This property together with the robust fermentation

41 kinetics inherited from the $S$. cerevisiae genome created yeast hybrid strains capable of

42 producing a crisp clean tasting lager that is now the most favoured alcohol-containing

43 beverage.

44 Genome analysis of S. pastorianus strains identified two distinct types, based on genome

45 content and chromosome composition (4, 12-17). Group I, or Saaz strains, are typically

46 triploid in DNA content, retaining all the parental S. eubayanus chromosomes but have lost

47 many S. cerevisiae chromosomes. This group includes several strains isolated from breweries

48 in Bavaria (Germany) and Bohemia (Czechia) and includes the strains CBS1538, CBS1513, 
49 and CBS1503 that were originally isolated in The Carlsberg Laboratory in the late $19^{\text {th }}$

50 century (18). The Group II, or Frohberg strains, include isolates from Dutch, Danish and

51 North American breweries and are mainly tetraploid in DNA content, containing

52 approximately $2 \mathrm{n}$ S. cerevisiae and 2n S. eubayanus genome content. Both groups display

53 chromosomal aneuploidy with chromosome numbers ranging from one to six $(19,20)$. In

54 addition to the parental chromosomes, S. pastorianus strains contain several hybrid

55 chromosomes containing both $S$. cerevisiae and S. eubayanus genes that resulted from

56 recombination, at precise locations, between the parental chromosomes. Some of the

57 recombination breakpoints are located within coding regions, creating a set of hybrid genes

58 unique to lager yeasts $(4,12,21,22)$.

59 Based on genome analysis, the current understanding of the origin and evolution of $S$.

60 pastorianus strains is that both Group I and II strains evolved from a common hybridisation

61 event between S. eubayanus and S. cerevisiae strains to generate a progenitor hybrid.

62 Subsequently, this progenitor strain underwent a second hybridisation event with a second $S$.

63 cerevisiae strain to generate Group II strains $(4,23)$. This model is supported by shared

64 recombination sites on hybrid chromosomes, evidence of Single Nucleotide Polymorphisms

65 (SNPs) in the S. cerevisiae genome of Group II strains and differences in telomeric regions in

66 Group I and II strains (23). The genome data is also consistent with a scenario in which both

67 groups emerged from a single hybridisation event between a diploid S. eubayanus strain and

68 a heterozygous S. cerevisiae diploid strain, with Group I strains experiencing a selective loss

69 of a significant proportion of the heterogeneous S. cerevisiae genome $(16,20,24)$. Both

70 groups then evolved independently with each undergoing further recombination events

71 between the sub-genomes $(3,20,22,24)$. Both groups have subsequently diverged to create

72 distinct sub-groups, each with their own unique physiological and biological properties. 
73 Fermentation analysis of Group I and II strains reveals that each group produces distinctive

74 aroma and flavour profiles (25).

75 The complex genome of $S$. pastorianus, containing orthologous alleles emanating from

76 different parental chromosomes, poses interesting questions regarding gene regulation and its

77 impact on the fermentation properties of the strains. Superimposed on the presence of

78 orthologous alleles are complexities of gene dosage due to the aneuploid nature of the

79 genomes. Aneuploidy has been shown to influence gene expression patterns in eukaryotic

80 cells (26-29). The presence of gene orthologues from two different parental Saccharomyces

81 species, together with copy number differences between the orthologues has the potential to

82 affect the cellular proteome and specifically the stoichiometry of $S$. cerevisiae and $S$.

83 eubayanus proteins within protein complexes (30). Furthermore, gene copy number

84 differences between the Group I and Group II strains may lead to differences in cellular

85 physiology and thus the fermentation properties of the two types of S. pastorianus.

86 Previous transcriptome analyses of $S$. pastorianus strains under fermentation conditions were

87 limited by technology and mainly focussed on the analysis of S. cerevisiae genes (31-34).

88 Such studies predated the discovery of $S$. eubayanus as a contributing parent to $S$.

89 pastorianus and advances in RNA sequencing technologies (35). More recent transcriptome

90 studies focussed on the analysis of sub-sets of genes with specific roles in fermentation or in

91 specific physiological conditions such as cold storage or responses to temperature (36-38).

92 Important findings regarding the gene expression of genes involved in maltose utilisation,

93 carbohydrate metabolism, glycerol mobilisation, anaerobiosis, and protein biosynthesis have

94 emerged from these studies, providing a road map for a more detailed transcriptome analysis

95 of these industrial strains.

96 Here, we analysed the transcriptomes of the Group I, CBS1538 and the Group II, WS 34/70

97 S. pastorianus strains and specifically examined the contribution of the two sub-genomes to 
98 the gene expression patterns under fermentations conditions. Using de novo genome

99 sequencing of these strains, we related gene expression patterns to gene copy numbers. We

100 show that the relative expression of S. cerevisiae and S. eubayanus orthologues is directly

101 correlated to gene copy number in the Group II strain. Gene copy number plays a smaller role

102 in gene expression patterns in the Group I strain, most likely due to the more limited genetic

103 redundancy. Despite the reduced S. cerevisiae content in the Group I strain, S. cerevisiae

104 orthologues contribute significantly to biochemical pathways upregulated during

105 fermentation, specifically to amino acid metabolism. Finally, comparison of the transcript

106 patterns in the Group I and II strains identified both common and unique gene expression

107 patterns during fermentation.

109 Results.

\section{Fermentation profiles of Group I and II strains.}

111 Two S. pastorianus strains, CBS1538 and WS 34/70, representative of Group I and Group II 112 strains respectively, were chosen for analysis. Both strains displayed similar fermentation 113 profiles in 10\% wort up to Day 4 but thereafter, strain WS 34/70 fermented faster than strain 114 CBS1538 and reached a lower final attenuation (Figure 1A). At the end of fermentations in

$1153 \mathrm{~L}$ tall tubes, the two strains produced similar volatile compound profiles with the exception 116 that ethyl butyrate and methionol levels were higher in WS 34/70, and ethyl hexanoate was 117 produced in higher levels in strain CBS1538 (Figure 1B). Similar volatile profiles were 118 obtained in small scale $(15 \mathrm{~mL})$ fermentations, the scale used for RNA extractions, with the 119 exception that higher levels of acetaldehyde were observed in both strains (data not shown). 
122 To compare the transcriptomes of Group I and Group II lager yeasts, RNA was extracted

123 from strains CBS1538 and WS 34/70 on Day 2 and Day 4 of small-scale fermentations in

$12410 \%$ wort. These time points were chosen as previous studies have shown that this is the

125 period of maximum metabolism during the fermentation (32). RNA was also extracted from

126 the same strains grown in minimal medium without amino acids to provide a baseline for

127 comparison. To map the transcripts, the genomes of CBS1538 and WS 34/70 isolates were

128 sequenced de novo and annotated using data from the annotated and fully assembled

129 reference genome S. pastorianus 1483 (Group II strain) as well as to a combined genome

130 assembled from the parental reference genomes $S$. cerevisiae and S. eubayanus. Both

131 approaches yielded highly similar results, however since the $S$. pastorianus 1483 genome

132 lacked some information for $S$. cerevisiae genes on chromosomes III and VII, the data from

133 mapping to the combined parental genomes was used for this analysis. Information from

134 genome sequencing and mapping of the two strains (Figure 2A, B) confirmed the absence of

135 S. cerevisiae chromosomes II, III, IV, IV, VII, VIII, XII, XIII, XV and XVI in CBS1538

136 (Figure 2A). Furthermore, we observed alterations in the copy number of chromosomes and

137 differences in hybrid chromosomes to the previously reported chromosome content of both

138 strains $(16,22,24)$ (Figure 2C). The estimated chromosome copy numbers were 48 and 76

139 for CBS1538 and WS 34/70 respectively.

141 Gene expression profiles of Group I and II strains during fermentation.

142 Transcripts were either assigned as S. cerevisiae (Sc) or S. eubayanus (Se) based on sequence

143 identity and chromosome assignment. A total of 11335 transcripts/Open Reading Frames

144 (ORFs) were detected for the WS 34/70 strain of which 5083 were assigned as S. eubayanus

145 and 6252 as $S$. cerevisiae (ratio Se:Sc $=0.81: 1$ ) (Supplemental Table 1). For the Group I

146 strain, a total of 6798 transcripts were detected, 5163 were $S$. eubayanus and 1635 were $S$. 
147 cerevisiae (ratio Se:Sc $=3.16: 1$ ). These values agree well with the previously determined

148 gene count for the two strains (39). We examined the differential gene expression (DEG) of

149 transcripts mapped to each strain in three different experimental conditions (minimal medium

150 without amino acids, wort Day 2 and wort Day 4) (Supplemental Tables 2-4). In the WS

$15134 / 70$ strain, the greatest number of genes displaying DEG was detected in the comparison of

152 cells grown in minimal medium and wort on Day 2 where $42 \%$ of transcripts were

153 differentially expressed, while $28 \%$ of genes were differentially expressed between growth in

154 minimal medium and in wort on Day 4. A much lower level of DEG was detected between

155 cells grown in wort on Days 2 and 4 where just $14 \%$ of genes were differentially expressed.

156 The pattern of DEG in CBS1538 was similar to that observed with the WS 34/70 strain with

$15732 \%$ of transcripts differentially expressed between growth in minimal medium and wort on

158 Day 2, 23\% between growth in minimal medium and wort on Day 4 and $16 \%$ between 159 growth in wort on Days 2 and 4.

160 As S. pastorianus contains both S. cerevisiae and S. eubayanus sub-genomes, we examined

161 the DEG patterns for $S$. cerevisiae or $S$. eubayanus alleles under the three different

162 experimental conditions. In the WS 34/70 strain, S. eubayanus genes are significantly over-

163 represented in the differentially upregulated gene pool under all three conditions but were

164 expressed at the expected ratio in the differentially downregulated gene pool (Figure 3A).

165 Interestingly, on Day 2 relative to Day 4 in wort, there are twice as many Se alleles

166 upregulated compared to Sc alleles. In the CBS1538 strain, Se alleles are overrepresented in

167 both the upregulated and downregulated gene pools in cells in wort on Day 2 relative to Day

1684 and in wort on Day 2 relative to minimal medium and in the downregulated gene pool in

169 wort on Day 4 relative to minimal medium.

170 The mean $\log _{2}$-fold change for $S$. cerevisiae and $S$. eubayanus alleles in both the WS $34 / 70$

171 and CBS1538 strains are extremely consistent under all conditions indicating that both Group 
172 I and Group II strains, and their Se and Sc sub-genomes, respond similarly to the

173 physiological conditions. The mean $\log _{2}$-fold change and the range of DEG on Days 2 and 4

174 wort was less than that observed for differential expression between minimum medium and

175 wort on Days 2 and 4 for both strains with the exception for down regulated genes on Days 2

176 and 4 in CBS1538 (Figure 3A and Supplemental Fig1).

177 We next examined the relationship of DEG under the three conditions in the two strains. As

178 might be expected, a large number of genes are differentially expressed between growth in

179 minimal medium and in wort on Days 2 and 4 in both the Group I and Group II strains

180 (Figure 3B). While fewer in number, there is an overlap in the genes differentially expressed

181 in wort Day 2 relative to Day 4 and in wort Day 2 relative to minimal medium. On the other

182 hand, there are no genes differentially expressed that are specific to wort Day 2 relative to

183 Day 4 and to wort Day 4 relative to minimal medium in WS $34 / 70$ and just 1 gene in this 184 category in CBS1538.

185 Only a small number of genes are commonly upregulated or downregulated respectively in 186 all three conditions in the Group I and the Group II strains (Figure 3B, Supplemental Table

187 5). The commonly upregulated gene pool in WS 34/70 $(n=210)$, contains 23 pairs of Sc and 188 Se orthologous alleles, including, ADH1, ADH5, PGK1, GPH1, HXK1, ENO1, that are 189 central to carbohydrate metabolism and ILV6, required for branched chain amino acid 190 biosynthesis. Likewise, both Sc and Se alleles of LEU4, LYS1 and SPD1 are upregulated in 191 all three conditions in CBS1538. Orthologous gene pairs are also observed in the commonly 192 downregulated gene in both strains (Supplemental Table 5).

194 Gene ontologies enriched in Group I and II strains under fermentation conditions.

195 Gene ontology analysis of the differentially expressed genes under the three conditions in the

196 Group I and Group II strains revealed an enrichment in both carbohydrate and amino acid 
197 metabolism on Day 2 of fermentation in the upregulated gene pool (Figure 4A). Genes

198 required for the utilisation of all major sugars including sucrose, fructose, galactose as well as

199 pentose sugars along with the genes required for glycolysis and pyruvate metabolism are all

200 upregulated in wort Day 2 relative to minimum medium and a subset of these genes are

201 upregulated on Day 2 relative to Day 4 and on Day 4 relative to minimum medium in the

202 Group II strain. Except for genes involved in starch and sucrose metabolism, the observed

203 gene set for carbohydrate metabolism in the Group II strain were not enriched in the Group I

204 strain (Figure 4A). Genes for the biosynthesis and metabolism of amino acids are enriched

205 during the fermentation in wort in both Group I and Group II strains (Figure 4A). In addition

206 to the enrichment of genes for these major metabolic activities in the two strains, we

207 observed differences in specific gene ontologies between the two strains, with genes involved

208 in glycerolipid metabolism, porphyrin metabolism, ABC transporters, peroxisome activity

209 and longevity upregulated in the Group II strain and not the Group I strain.

210 Genes involved in nucleic acid metabolism, DNA repair and DNA replication are

211 downregulated under fermentation conditions and on Day 2 relative to Day 4 in wort in the

212 Group II strain with a subset of these genes similarly regulated in the Group I strain (Figure

213 4B). Likewise, genes involved in major anabolic pathways such as protein synthesis

214 including genes required for ribosome biogenesis and rRNA, mRNA and tRNA processing

215 are downregulated in wort and on Day 2 relative to Day 4 in both strains. Interestingly, a set

216 of genes involved in fatty acid, glycerophospholipid and steroid metabolism, required for the

217 production of membrane components, are uniquely downregulated in the Group I strain

218 (Figure 4B).

219 We examined the contribution of the Sc and Se sub-genomes to these enriched pathways. Se

220 and Sc alleles were observed to contribute at the expected Se:Sc ratio or in a higher

221 proportion than expected to the differentially regulated gene pool (Figure 4A, B). Se alleles 
222 contribute in a higher proportion than expected on Days 2 and 4 of fermentation in the Group

223 II strain (Figure 4A). Conversely, we observed that Sc alleles contribute in a greater

224 proportion than expected to amino acid metabolism in the Group I strain under all three

225 conditions (Figure 4A). For some specific pathways, we observed that Se alleles accounted

226 for all the differentially regulated genes, for example Se alleles are used exclusively in

227 histidine metabolism on Day 2 relative to Day 4 in the Group II strain and to phagosome

228 associated genes in wort on Day 2 relative to Day 4 and to sphingolipid, butanoate and

229 thiamine metabolism on Day 4 relative to minimum medium and to phagosome activity on

230 Day 2 relative to Day 4 in wort in the Group I strain (Figure 4A).

231 For the downregulated genes, we observe that Sc alleles contribute to gene ontologies at the

232 expected ratio, and in some pathways, in a higher proportion than expected to the ratio of

233 Sc:Se for the whole genome in both strains while Se alleles exclusively contribute to three

234 categories, namely one carbon pool by folate, fatty acid and steroid biosynthesis in the Group

235 I strain (Figure 4B).

237 The effect of gene dosage on gene expression profiles.

238 As $S$. pastorianus strains are aneuploid in nature and have different copy numbers of $S$.

239 cerevisiae and S. eubayanus chromosomes, we were interested to determine if gene

240 expression profiles were influenced by the copy number of Sc and Se alleles. The copy

241 number for each Sc and Se gene was calculated from the sequence coverage from the de novo

242 genome sequencing. The DEG of Sc and Se orthologues within each strain was determined

243 for the three experimental conditions and was correlated to the Sc:Se gene copy number ratio.

244 The data for Day 2 vs min is shown in Figure 5. Sc and Se orthologues are present in varying

245 ratios in the Group II strain (Figure 5A). The analysis of some $1555 \mathrm{Sc}$ and Se orthologues

246 indicates that the DEG of Sc and Se alleles was positively correlated to the ratio of the copy 
247 number of Sc:Se alleles (Figure $5 \mathrm{~A}, \mathrm{~B}, \mathrm{p}<0.0001, \mathrm{R}^{2}=0.90$ ). As the Sc copy number

248 increases, so too does the levels of Sc transcripts and vice versa for Se alleles. At an Sc:Se

249 ratio of $1: 2$ and 3:4, Se transcripts predominate while at ratios, 3:1, 4:1 and 5:1 Sc alleles

250 predominate. Interestingly, at a 1:1 ratio, Se alleles predominate in the Group II strain while

251 at 3:2, 2:1 there is almost equal levels of both transcripts. The relationship between the DEG

252 of Sc and Se orthologues and the ratio of gene copy number is observed for genes on both

253 parental and hybrid chromosomes as exemplified in Figure 5C for chromosome X. The WS

$25434 / 70$ strain has four copies of chromosome X, one Sc, one Se and 2 hybrid chromosomes

255 (Sc/Se) with the recombination point occurring at the gene THD2 (YJR009C) (Figure 2C).

256 The ratio of Sc:Se to the left of $T D H 2$ is 3:1 and to the right 1:1 (Figure 2C). For genes to the

257 left of $T D H 2$, expression of Sc alleles predominates due to the higher Sc copy number while

258 to the right, Se alleles predominate despite the equal copy number (Figure 5C). The

259 correlation of DEG patterns to orthologue gene copy number ratio was observed in all three

260 experimental conditions (data not shown).

261 The analysis of the gene expression of Sc and Se orthologues in the Group I strain presented

262 a different scenario. Firstly, due to the reduction of the Sc genome content in the Group I

263 strain, the number of orthologues is much smaller $(n=237)$. Secondly, the variations in copy

264 numbers are much smaller, with most orthologues present in a ratio of Sc:Se, 1:2 (Figure

265 5D). There are just two alleles present in a 2:3 and one at a 2:1 ratio (data not shown).

266 Surprisingly, we see a different expression pattern to what was observed in Group II; at the

267 Sc:Se 1:2 ratio, a majority of Sc alleles display higher transcript levels than the orthologous

268 Se alleles, although the reverse expression pattern is evident for some orthologues.

270 Comparison of the transcriptomes of Group I and Group II strains. 
271 To directly compare the steady state mRNA landscapes in the Group I and II strains, we

272 generated a consolidated transcriptome for each strain by obtaining the sum of Sc and Se

273 allele transcripts encoded for each gene (Supplemental Table 6). We compared the expression

274 of this gene set in the Group I and II strains under the three different experimental conditions

275 (Figure 6 and Supplemental Table 7). There is a high degree of overlap between the

276 differentially expressed gene pool in both strains suggesting that both strains respond

277 similarly to the environmental conditions (Figure 6A). Growth in minimal medium invoked

278 the greatest condition specific DEG between the two strains but a substantial number of

279 genes were also specifically expressed in wort on Day 2 and Day 4 respectively. We

280 identified 310 Group I-specific genes that are upregulated in all three conditions and likewise

281299 Group II-specific genes. The list of condition specific and Group-specific genes is shown

282 in Supplemental Table 8. An analysis of gene ontologies associated with the differentially

283 expressed gene set reveals differences between the Group I and II strains (Figure 6B). Firstly,

284 we observed that genes associated with ribosome biosynthesis are enriched on Day 2 and 4 in

285 wort in the Group II strains while genes associated with metabolism of certain amino acid,

286 pentose phosphate pathway and oxidative phosphorylation are also upregulated on Day 2 in

287 this strain. Genes associated with DNA repair and protein processing and transport are

288 upregulated in minimal medium. Conversely, genes associated with membrane biosynthesis,

289 sugar metabolism and amino acid metabolism are upregulated in minimal medium in the

290 Group I strain, and a subset of these genes are also upregulated on Day 4 in wort.

291 Surprisingly, there are no gene ontologies enriched on Day 2 in the Group I strain (Figure

$2926 B)$.

293 As the gene copy number for the sum of gene orthologue transcripts still varied between

294 Group I and II strains and was shown to influence gene expression profiles within a strain, we

295 looked to see if gene copy number influences the DEG observed between the Group I and 
296 Group II strain. To do this, we compared the $\log _{2}$ fold changes in gene expression between

297 the two strains to the ratio of the total gene copy number (sum of all Se and Sc alleles) for

298 each gene. The data for gene expression on Day 2 in wort is shown in Figure 6C. There is a

299 significant positive correlation between $\log _{2}$ fold change and ratio of total gene copy number

300 between the two strains $\left(\mathrm{p},<0.005, \mathrm{R}^{2}=0.8\right)$. This correlation was observed across all three

301 experimental conditions (data not shown). Thus, as observed for gene expression within a

302 strain, gene dosage plays a significant role in the DEG between the Group I and Group II

303 strains.

304 Discussion.

305 The origin and evolution of lager yeasts are widely debated, and several hypotheses have

306 been proposed to account for the divergence in chromosome composition and copy number

307 between the Group I and II strains $(20,23,24)$. The major distinction between the two groups

308 is the loss a significant portion of the S. cerevisiae sub-genome through the loss of whole

309 chromosomes in the Group I strains. In addition, differences in chromosome composition,

310 copy number and the number and type of hybrid chromosomes exist between strains within

311 each group. Despite these differences, strains from both groups ferment sugars and produce

312 aromatic beers, with individual strains displaying variations in the final aromatic volatile

313 profile (25). A major difference between Group I and II strains lies in the ability to ferment

314 maltotriose. While all Group II strains can uptake and ferment maltotriose, only a subset of

315 Group I strains have the necessary transporters to import this trisaccharide (40-42). The

316 Group I strain used in this study, CBS1538, does not ferment maltotriose. We confirm here

317 the superior fermentation rates of the Group II strains and show that the two strains produce

318 unique flavour profiles. The two strains consume sugars at the same rate up to Day 4 and

319 thereafter the Group II strain fermented at a faster rate. WS 34/70 produces more isoamyl

320 acetate than CBS1538, surpassing olfactory thresholds. This ester comes from the 
321 esterification of the higher alcohol isoamyl alcohol and imparts banana-like aromas, a

322 desirable compound in beer. The WS34/70 also produced higher levels of ethyl butyrate

323 while in contrast, CBS1538 overproduces ethyl hexanoate. Both esters contribute to the

324 tropical fruit aroma in beer $(43,44)$.

325 We were interested in understanding how the two sub-genomes, present in the strains,

326 contribute to the overall transcriptome during fermentation and furthermore what are the

327 consequences for the reduction in the $S$. cerevisiae sub-genome to the gene expression

328 patterns between the Group I and II strains. The complex genomes of lager yeasts pose

329 several challenges to the analysis of the transcriptomes of these strains. At present, just one $S$.

330 pastorianus Group II genome, that of strain CBS1483, has been fully annotated and

331 assembled into chromosomes and this serves as the reference genome for S. pastorianus at

332 NCBI (20), although up to 16 annotated genomes are also available

333 https://www.ncbi.nlm.nih.gov/genome/browse/\#!/eukaryotes/342/ . Due to differences in

334 chromosome copy numbers, and chromosomal rearrangements, the reference genome may

335 not be the ideal genome for transcript mapping, for example, it differs from the two strains,

336 WS 34/70 and CBS 1538, used in this study, as it lacks hybrid chromosomes III and VII.

337 There are also chromosome copy number differences between the strains used here and the 338 reference strain.

339 To avoid such issues, we re-sequenced the WS 34/70 and CBS1538 strains and used the

340 combination of the parental strains $S$. cerevisiae and S. eubayanus genomes to map the

341 transcriptomes. The copy number for each annotated gene was determined from the sequence

342 coverage depth from de novo sequencing.

343 We noted some differences in the chromosome composition of both WS 34/70 and CBS1538

344 to the published data $(16,24,45)$. Specifically, we noted one extra copy of chromosomes Sc

345 III, VII, XIV, and XVI in relation to the most recently published WS 34/70 sequence (24). 
346 Additionally, we reconfirm the presence of a hybrid chromosome, ScVIII/SeVIII,

347 recombining at YHR165C, that was previously identified in the WS 34/70 strain and in

348 another Group II strain 7012 by our group $(12,21)$ but which is not documented in other

349 published sequences $(16,24)$. For the CBS1538, we noted just two copy number differences,

350 an extra copy of both Se VI and SeVIII. Chromosome copy number differences are also

351 noted between published genomes for WS 34/70 (16, 24, 45). The differences in copy

352 numbers between studies may arise from differences in methods used to determine sequence

353 coverage depth or may reflect genuine differences between strain isolates. The lager yeast

354 strains emerged just some 500-600 years ago and thus may still be experiencing genomic flux

355 in this early stage of evolution. We previously showed that the genomes of lager yeasts are

356 dynamic and can undergo chromosome rearrangements to produce new hybrid chromosomes

357 as well as chromosome copy number changes following exposure to stress such as high

358 temperatures (46). Furthermore, fermentations carried out in high specific gravity wort $\left(22^{\circ} \mathrm{P}\right)$

359 at an ambient temperature of $20^{\circ} \mathrm{C}$, which is higher than that used for routine fermentations

$360\left(13^{\circ} \mathrm{C}\right)$, led to chromosome copy number changes in a single round of fermentation (46).

361 Thus, differences in propagation and culturing of $S$. pastorianus strains may contribute to the

362 differences observed between strain isolates.

363 The analysis of the gene expression patterns reveals that the Group I and Group II strains

364 respond similarly to the physiological conditions imposed with both strains showing similar

365 mean $\log _{2}$ fold changes under all conditions. Interestingly, we observed that the Sc and Se

366 sub-genomes are differentially utilised under the different physiological conditions with Se

367 alleles contributing significantly to gene expression on Day 2 of fermentation in both strains

368 and on Day 4 in the Group II strain.

369 Gene ontology analysis revealed the upregulation of pathways associated with carbohydrate

370 and amino acid metabolism on Day 2 of fermentation although there was less enrichment of 
371 genes associated with sugar metabolism in the Group I strain. The upregulation of

372 carbohydrate and amino acid metabolism in the early stages of fermentation is consistent with

373 what is known about the overall metabolic activity of yeast during fermentation. Cells

374 undergo 1-2 doublings during the first three days of fermentation and thereafter, cell numbers

375 remain unchanged or slightly decrease. The lack of representation of genes associated with

376 carbohydrate metabolism in the Group I strain, except for those associated with starch and

377 sucrose metabolism, distinguishes the two strains and appears to reflect differences in

378 metabolic activity. As overall sugar consumption is similar in the two strains on Day 2, it is

379 possible genes associated with carbohydrate metabolism in the Group I strains are induced

380 but did not reach the cut off threshold for analysis. It does not appear that sugar metabolism

381 is slowed down in this strain as otherwise we may have expected to see such genes

382 upregulated on Day 4. The observed differences in carbohydrate metabolism may reflect the

383 previously noted differences in the types and copy numbers of genes encoding for maltose

384 and maltotriose transporters between the two strains (40-42).

385 The upregulation of genes associated with amino acid metabolism is significant as the

386 secondary metabolites associated with flavour such as higher alcohols and esters are

387 produced from the catabolism of amino acids. The gene ontology also revealed differences in

388 metabolism between the two strains. For example, genes associated with methane, butanoate

389 and sulphur metabolism, which can also contribute to flavour profiles, are upregulated in

390 Group II but not in Group I.

391 Gene ontology analysis confirm the over representation of Se alleles in the upregulated genes

392 in the Group II strain and additionally identified specific pathways where either Sc or Se

393 alleles are exclusively enriched. Considering the reduced Sc content in the Group I strain, it is

394 surprising that Sc alleles appear to be over-represented in genes enriched in several pathways.

395 Specifically, we see Sc alleles contributing to Arginine and Lysine biosynthesis and Tyrosine 
396 metabolism at a higher level than expected from the Sc:Se ratio for the whole genome. We

397 also observed that Sc alleles are more likely to be downregulated than Se alleles in both

398 Group I and Group II strains however specific pathways here also contain exclusively Se

399 alleles.

400 Previous studies in the haploid S. cerevisiae showed that the steady state levels of mRNA

401 transcripts are tightly controlled to maintain homeostasis. Disruption of mRNA turnover, for

402 example by deletion of the major 5' to 3' exonuclease $X R N 1$, is compensated by changes to

403 RNA polymerase II transcription to restore homeostasis $(47,48)$. Conversely, increased

404 transcription rates resulting from increased gene copy number lead to compensatory changes

405 in mRNA turnover to preserve the expected steady state levels of mRNAs (49). However, a

406 recent analysis of laboratory and wild S. cerevisiae strains revealed no evidence of dosage

407 compensation and instead described a direct correlation between gene copy number and gene

408 expression levels (50).

409 Here we show that the aneuploid nature of the S. pastorianus genomes directly contributes to

410 the gene expression patterns during fermentation. We observed a positive correlation

411 between orthologue copy number and the steady state levels of orthologue transcripts in the

412 Group II strain: as the copy number of Sc or Se genes increases so too does the associated

413 levels of transcripts. Interestingly, when the orthologue gene copy number is 1:1, we

414 observed that the Se alleles showed higher levels of transcripts than the Sc alleles in all

415 conditions tested. Surprisingly, the pattern of usage of orthologues is different in the Group I

416 strain. While there is less variation in the ratios of Sc:Se genes with most orthologues present

417 in a 1:2 ratio, here we observed that Sc orthologues produce higher levels of transcripts than

418 the Se counterpart although there is a greater variation in the distribution of gene expression

419 patterns amongst orthologues at this ratio in the Group I strain. At the same ratio in the Group

420 II strain, Se alleles predominate. The over-representation of $S$. cerevisiae alleles in some 
421 biochemical processes such as amino acid metabolism may explain the selective retention of

422 S. cerevisiae chromosomes in the Group I strain as there may have been a selective

423 evolutionary pressure to retain specific genes required during fermentation.

424 The S. eubayanus parent of the lager yeasts is a cryotolerant strain and thus it might be 425 hypothesised that Se alleles will be favoured during the fermentations which are carried out 426 at $13^{\circ} \mathrm{C}$. We did observe a greater contribution of Se alleles to the transcriptome in the Group 427 II strain during fermentation conditions, however, it is not universal, and this contribution is 428 tempered by gene copy number effects. A transcriptome analysis for the Group I strain, 429 CBS1513, had also observed an overrepresentation of S. eubayanus when cultures were 430 grown at low temperatures (38).

431 To compare the overall steady state levels of protein encoding transcripts in the Group I and 432 II strains, we created combined transcriptomes for each strain by summing the Sc and $\mathrm{Se}$ 433 transcripts that encode for the same protein. Using this approach, we observed that that 434 overall transcription patterns were similar between the two strains indicating that despite the 435 significant differences at a genome level and pathways of evolution, the two lager yeast 436 groups display similar transcription patterns in fermentation conditions. Nevertheless, gene 437 ontology analysis identified some unique transcription patterns. Consistent with what was 438 observed for the differential expression of Sc and Se alleles within a strain, the gene 439 expression patterns in both strains were significantly influenced by gene copy number.

440 Thus, our analysis of the expression of gene orthologues within a strain and between strains 441 indicates that the aneuploid genomes of the lager yeasts create complex patterns of gene 442 expression during fermentation and that gene copy number plays a crucial role in the gene 443 expression patterns both within a strain and between strains. 
446 Yeast strains and growth conditions. The Group I strain CBS 1538 was obtained from the

447 Collection de Levures d'Interet Biotechnologique, Paris, France and Group II strain

448 Weinstephan 34/70 was kindly supplied by Dr. Jurgen Wendland, Geisenheim Hoch

449 Universitat, Germany. For propagation, strains were grown in YPDM medium (1\% (w/v)

450 yeast extract, $2 \%(\mathrm{w} / \mathrm{v})$ peptone and $1-2 \%(\mathrm{w} / \mathrm{v})$ of both dextrose and maltose at $20-25^{\circ} \mathrm{C}$;

$4512 \%$ YPDM contains $1 \%$ dextrose and $1 \%$ maltose.

452 For RNA extractions, cells were grown in 2\% YPDM overnight and were then washed with 453 sterile water and inoculated at a cell density of $1 \times 10^{6}$ cells $/ \mathrm{mL}$ into $60 \mathrm{~mL}$ of minimal 454 medium $(0.17 \%$ Yeast Nitrogen Base w/o amino acids and ammonium salts supplemented 455 with $1 \%$ of dextrose and $1 \%$ of maltose, and $0.5 \%(\mathrm{NH} 4)_{2} \mathrm{SO}_{4}$ as a nitrogen source). The 456 cultures were grown at $20^{\circ} \mathrm{C}$, in triplicate, and cells were harvested in the exponential phase.

457 Small-scale fermentations (10mL) and large-scale fermentations (2L) were carried out in $45810 \%$ wort containing $1 \mathrm{mM} \mathrm{ZnSO}_{4}$ (Spraymalt, Brewferm, Amber 18EBC, Brouland, 459 Belgium) in $15 \mathrm{~mL}$ glass test tubes or $3 \mathrm{~L}$ tall tubes in triplicate or duplicate respectively.

460 Cells were first propagated in $200 \mathrm{~mL}$ of $4 \%$ YPDM at $25^{\circ} \mathrm{C}$ for two days and were pitched at a 461 cell density of $1.5 \times 10^{7} / \mathrm{mL}$. The tubes were fitted with a water trap airlock attached to a bung 462 and incubated at $13^{\circ} \mathrm{C}$ at a $45^{\circ}$ angle without shaking. The specific gravity of the wort was 463 measured at the start of fermentation and at intervals throughout the fermentation using a 464 refractometer (HANNA, Romania).

466 Flavour Analysis.

467 Analysis of volatile compounds in wort at the end of fermentation were conducted at The 468 Research and Innovation Centre, Edmund Mach Foundation S.Michele all'Adige, Italy as 469 previously described (51). Briefly, $2.5 \mathrm{~mL}$ of the samples in $20 \mathrm{~mL}$ vials were supplemented 470 with sodium chloride to a final concentration of $40 \mathrm{mg} / \mathrm{mL}$ and $25 \mu \mathrm{L}$ of the 2-octanol as the 
471 internal standard (final concentration $200 \mu \mathrm{g} / \mathrm{L}$ ). All samples were incubated for $10 \mathrm{~min}$ at

$47240^{\circ} \mathrm{C}$, then the volatile compounds were collected on a divinylbenzene / carboxen /

473 polydimethylsiloxane fibre (DVB-CAR-PDMS) coating 50/30 $\mu \mathrm{m}$, and 2-cm length SPME

474 fibre purchased from Supelco (Sigma Aldrich, Milan, Italy) for $40 \mathrm{~min}$. GC analysis was

475 performed on a Trace GC Ultra gas chromatograph coupled with a TSQ Quantum Tandem

476 mass spectrometer (Thermo Electron Corporation, USA) (51). Identification of compounds

477 was based on comparison with a mass spectral database (NIST version 2.0) and with

478 reference standards when available. The relative amount of each volatile was expressed as

$479 \mu \mathrm{g} / \mathrm{L}$ of 2-octanol (52).

480 DNA extraction, de novo genome sequencing and copy number variation analysis.

481 DNA extraction was carried out following the protocol described by (53). Briefly, yeast cells

482 were grown in an overnight culture of $5 \mathrm{ml}$ of $2 \%$ YPDM. Cells were pelleted and washed

483 with distilled water and centrifuged. The pellet was resuspended in $500 \mu \mathrm{L}$ of $0.9 \mathrm{M}$ sorbitol

484 and $0.1 \mathrm{M}$ EDTA, $\mathrm{pH} 7.5$ and $30 \mu \mathrm{L}$ of a solution of lyticase $(1.5 \mathrm{mg} / \mathrm{mL})$ was added and

485 incubated for 20-40 min. After this, spheroplasts were recovered centrifuged and resuspended

486 in $500 \mu \mathrm{L}$ of $50 \mathrm{mM}$ Tris $\mathrm{HCl}$ and $20 \mathrm{mM}$ EDTA, pH 7.4 and $13 \mu \mathrm{L}$ of $10 \%$ SDS. Samples

487 were incubated for $65^{\circ} \mathrm{C}$ for $5-10 \mathrm{~min} .200 \mu \mathrm{L}$ of $5 \mathrm{M}$ potassium acetate were added and

488 tubes were incubated on ice for $10 \mathrm{~min}$. After centrifugation, $700 \mu \mathrm{L}$ of $100 \%$ isopropanol

489 was added to the supernatant, the samples incubated at room temperature for $10 \mathrm{~min}$ and then

490 recentrifuged. The pelleted DNA was washed twice with $70 \%$ ethanol, air-dried at room

491 temperature, and resuspended in $50 \mu \mathrm{L}$ of water.

492 Total DNA was treated with RNAse A $(10 \mu \mathrm{g} / \mathrm{mL})$ for $30 \mathrm{~min}$ at $37^{\circ} \mathrm{C}$. DNA was precipitated

493 and resuspended in $50 \mu \mathrm{L}$ of water. The quality of the DNA was determined by agarose gel

494 electrophoresis and by determining the $\mathrm{OD}_{260}: \mathrm{OD}_{280}$ ratio using a Nanodrop 
495 spectrophotometer (Epoch 2, Agilent BioTek). De novo genome sequencing was carried out

496 by Novogene (www.novogene.com) with Illumina technology on paired-end reads (150bp).

497 After trimming paired-end reads with trim_galore (version 0.6.6, calling cutadapt version

498 2.10), the reads were mapped with bowtie2 (version 2.4.2) $(54,55)$ to the parental genomes

499 of $\quad$ S. pastorianus CBS1483, (assembly ASM1102231v1)

500 https://www.ncbi.nlm.nih.gov/genome/?term=Saccharomyces+pastorianus, or to a combined

501 genome of $S$. cerevisiae and S. eubayanus derived from S. cerevisiae S288C, assembly R64

502 https://www.ncbi.nlm.nih.gov/genome/?term=Saccharomyces+cerevisiae and S. eubayanus

503 (SEU3.0) https://www.ncbi.nlm.nih.gov/genome/?term=Saccharomyces+eubayanus.

504 In addition to the '--fast-local' presets, parameters were set to limit the insert size to lengths

505 between 200 and 1000 basepairs. Mapped reads were filtered through samtools (version 1.11)

506 to exclude reads that were not mapped in pairs and were not the primary alignment (-F 268)

507 and to include only reads with high mapping quality (-q 44).

508 For hybrid chromosomes, genes were assigned as S. cerevisiae or S. eubayanus based on their

509 location relevant to known recombination sites and their \% identity to the parental genomes.

510 Reads from de novo DNA sequencing, mapped against the combined genomes of the

511 reference strains of S. cerevisiae and S. eubayanus were transformed into sorted BAM files

512 using samtools and the data was extracted as reads/500 bp and then normalized by the size of

513 the library (total number of reads) to give an estimated chromosome copy number (56).

515 RNA extraction and Sequencing

516 RNA extraction was carried out following the protocol previously described (57) but with

517 some modifications. Cells were resuspended in $400 \mu \mathrm{L}$ of buffer containing $50 \mathrm{mM}$ of

518 NaOAc, pH 5.2, $10 \mathrm{mM}$ EDTA pH 8.0 and $40 \mu \mathrm{L}$ of $10 \%$ SDS, $400 \mu \mathrm{L}$ of Phenol-

519 Chloroform-Isoamyl alcohol (PCA; 25:24:10), $\mathrm{pH} 4.2$, and $100 \mu \mathrm{L}$ of acid washed glass 
520 beads were added. The tubes were placed in a heat block at $65^{\circ} \mathrm{C}$ for $10 \mathrm{~min}$ and vortexed

521 intermittently. The samples were placed on ice for $5 \mathrm{~min}$. After centrifugation, the

522 supernatant was re-extracted with PCA, $\mathrm{pH} 4.2$ and then once with chloroform. Nucleic acids

523 in the supernatant were precipitated, washed twice with $70 \%$ ethanol and then resuspended in

$524100 \mu \mathrm{L}$ RNase-free $\mathrm{H}_{2} \mathrm{O}$. The RNA concentration was measured using a Nanodrop

525 spectrophotometer and RNA integrity was checked by Agilent 2100 Bioanalyzer as per

526 manufacturer's instructions.

527 RNA sequencing was conducted on cDNA libraries using Illumina technology at the

528 Genomic Technologies Core Facility at the University of Manchester. As a reference genome

529 for the mapping, the combined S. cerevisiae and S. eubayanus genomes were used but with

530 variations as present in the re-sequenced CBS1538 and WS 34/70 strains. For that, consensus

531 Fasta sequences were generated from the WGS data mapped to $S$. cerevisiae and $S$.

532 eubayanus through an in-house Perl script. To transfer gene annotation from those genomes,

533 insertions and deletions were ignored and only nucleotide replacements were taken into

534 account. Approximately 88k and 126k reads were unmapped for CBS1538 for WS 34/70

535 respectively. The paired RNA-Seq data were mapped against these strain-specific reference

536 genomes using STAR (58). Multimapping reads are dealt with through the

537 outSAMmultNmax parameter set to 1 and outMultimapperOrder set to 'random'.

538 Additionally, only the highest quality alignments were kept by filtering with samtools (-q

539 255). To aggregate reads counts, the tool "featureCounts" was used on the mapped data

540 together with the gene annotation from the S. cerevisiae and S. eubayanus genome (59). With

541 the -B and the -P options set, only read counts that have both ends aligned within a distance

542 of 50-600 basepairs were considered.

543 Read counts from the RNA mapping were uploaded into iDEP9.1 (60). Data were

544 transformed for clustering and PCA by EdgeR using a minimum CPM of 0.5 in 1 library and 
545 adding a pseudocount of 4. PCA analysis was used to check the reproducibility of replicates.

546 Outlier replicates were removed and data reprocessed. Differentially Expressed Genes (DEG)

547 were calculated using DESeq2 with a False Discovery Rate (FDR) cutoff of 0.05 and a

548 minimum $\log _{2}$ fold change of $\geq 1$ or $\leq-1$. Venn diagrams were made using the online tool

549 (http://bioinformatics.psb.ugent.be/). The copy number for each gene was extracted from the

550 reads coverage of the de novo genome sequencing of the $S$. pastorianus strains.

551 Enrichment of DEG was carried out using ClueGO (61). Parameters used on ClueGo were set

552 as follows: Saccharomyces cerevisiae S228C was used as a Load Maker List. KEGG

553 Ontology was used for the analysis with the following filters, p-value less than 0.05 ,

554 minimum number of four genes per pathway, pathways containing more than $25 \%$ of the

555 genes regulated, Kappa score was set at 0.4. A two-sided hypergeometric test and a

556 Benjamini-Hochberg $\mathrm{pV}$ correction was applied. S. eubayanus-like allele names were

557 translated into S. cerevisiae gene names for ClueGo analysis.

558 A combined transcriptome of $S$. pastorianus genes was generated by summing the reads for

559 S. cerevisiae and S. eubayanus orthologues using the consolidate function in Excel.

560 Unmatched genes were retained in the combined transcriptome with reads mapped

561 respectively to the $S$. cerevisiae or S. eubayanus reference genomes. The combined genome

562 contained 7568 unique gene ids. The copy number for each gene was extracted from the

563 reads coverage of the de novo genome sequencing of the $S$. pastorianus strains and were

564 summed for the matched orthologues to give a total gene copy number for each gene id.

566 Statistics.

567 False discoveries rates for DEG and gene ontologies were determined using the Benjamini-

568 Hochberg correction. Chi-squared tests were used to determine the enrichment of genes from

569 sub-genomes under the different physiological conditions used. 
571 Acknowledgements. We thank Penghan Zhang, Silvia Carlin and Urska Vrhovsek for 572 assistance with GC/MS analysis.

\section{References}

1. Hornsey IS. A history of beer and brewing. Cambridge, UK: The Royal Society of 577 Chemistry; 2003.

578 2. Barnett JA. A history of research on yeasts 2: Louis Pasteur and his contemporaries,

3. Monerawela C, Bond U. Brewing up a storm: The genomes of lager yeasts and how they evolved. Biotechnol Adv. 2017;35(4):512-9.

4. Dunn B, Sherlock G. Reconstruction of the genome origins and evolution of the hybrid lager yeast Saccharomyces pastorianus. Genome Res. 2008;18(10):1610-23.

5. Libkind D, Hittinger C, Valério E, Gonçalves C, Dover J, Johnston M, et al. Microbe domestication and the identification of the wild genetic stock of lager-brewing yeast. Proceedings of the National Academy of Sciences of the United States of America. 2011;108(35):14539-44.

6. Peris D, Langdon QK, Moriarty RV, Sylvester K, Bontrager M, Charron G, et al. Complex Ancestries of Lager-Brewing Hybrids Were Shaped by Standing Variation in the Wild Yeast Saccharomyces eubayanus. PLoS Genet. 2016;12(7):e1006155.

7. Langdon QK, Peris D, Eizaguirre JI, Opulente DA, Buh KV, Sylvester K, et al. Postglacial migration shaped the genomic diversity and global distribution of the wild ancestor of lager-brewing hybrids. PLoS Genet. 2020;16(4):e1008680.

8. Bing J, Han P-J, Liu W-Q, Wang Q-M, Feng-Yan B. Evidence for a Far East Asian origin of lager beer yeast. Current Biology. 2014;24(10).

9. Eizaguirre JI, Peris D, Rodriguez ME, Lopes CA, De Los Rios P, Hittinger CT, et al. Phylogeography of the wild Lager-brewing ancestor (Saccharomyces eubayanus) in Patagonia. Environ Microbiol. 2018;20(10):3732-43.

10. Alsammar HF, Naseeb S, Brancia LB, Gilman RT, Wang P, Delneri D. Targeted metagenomics approach to capture the biodiversity of Saccharomyces genus in wild environments. Environ Microbiol Rep. 2019;11(2):206-14.

11. Krogerus K, Seppanen-Laakso T, Castillo S, Gibson B. Inheritance of brewingrelevant phenotypes in constructed Saccharomyces cerevisiae x Saccharomyces eubayanus hybrids. Microb Cell Fact. 2017;16(1):66.

12. Bond U, Neal C, Donnelly D, James T. Aneuploidy and copy number breakpoints in the genome of lager yeasts mapped by microarray hybridisation. Current genetics. 2004;45(6):360-70.

13. Kodama Y, Kielland-Brandt MC, Hansen J. Lager brewing yeast. In: Sunnerhagen P, Piskur J, editors. Topics in Current Genetics. Topics in Current Genetics. 15. Berlin, Heidelberg: Springer; 2006. p. 145-64.

14. Nakao Y, Kanamori T, Itoh T, Kodama Y, Rainieri S, Nakamura N, et al. Genome sequence of the lager brewing yeast, an interspecies hybrid. DNA Research 2009;16(2):115-29. 
614 15. Walther A, Hesselbart A, Wendland J. Genome Sequence of Saccharomyces

615

616

617

618

619

620

621

622

623

624

625

626

627

628

629

630

631

632

633

634

635

636

637

638

639

640

641

642

643

644

645

646

647

648

649

650

651

652

653

654

655

656

657

658

659

660

661

662

663

carlsbergensis, the World's First Pure Culture Lager Yeast. G3 (Bethesda, Md). 2014;4(5):783-93.

16. Wendland J. Lager yeast comes of age. Eukaryotic Cell. 2014;13(10):1256-65.

17. Hewitt S, Donaldson I, Lovell S, Delneri D. Sequencing and characterisation of rearrangements in three $\mathrm{S}$. pastorianus strains reveals the presence of chimeric genes and gives evidence of breakpoint reuse. arXiv preprint arXiv: .... 2013.

18. Barnett JA, Lichtenthaler FW. A history of research on yeasts 3: Emil Fischer, Eduard Buchner and their contemporaries, 1880-1900. Yeast. 2001;18(4):363-88.

19. Monerawela C, Bond U. The hybrid genomes of Saccharomyces pastorianus: A current perspective. Yeast. 2018;35(1):39-50.

20. Salazar AN, Gorter de Vries AR, van den Broek M, Brouwers N, de la Torre Cortes P, Kuijpers NGA, et al. Chromosome level assembly and comparative genome analysis confirm lager-brewing yeasts originated from a single hybridization. BMC Genomics. 2019;20(1):916.

21. Monerawela C, Bond U. Recombination Sites on Hybrid Chromosomes in S. pastorianus share Common Sequence Motifs and define a Complex Evolutionary Relationship between Group I and II Lager Yeasts. FEMS Yeast Research. 2017; 17(5):fox047.

22. Hewitt SK, Donaldson IJ, Lovell SC, Delneri D. Sequencing and characterisation of rearrangements in three $\mathrm{S}$. pastorianus strains reveals the presence of chimeric genes and gives evidence of breakpoint reuse. PLoS One. 2014;9(3):e92203.

23. Monerawela C, James TC, Wolfe KH, Bond U. Loss of lager specific genes and subtelomeric regions define two different Saccharomyces cerevisiae lineages for Saccharomyces pastorianus Group I and II strains. FEMS Yeast Res. 2015;15(2).

24. Okuno M, Kajitani R, Ryusui R, Morimoto H, Kodama Y, Itoh T. Next-generation sequencing analysis of lager brewing yeast strains reveals the evolutionary history of interspecies hybridization. DNA Research. 2016;23(1):67-80.

25. Gibson BR, Storgards E, Krogerus K, Vidgren V. Comparative physiology and fermentation performance of Saaz and Frohberg lager yeast strains and the parental species Saccharomyces eubayanus. Yeast. 2013;30(7):255-66.

26. Ried T, Meijer G, Harrison DJ, Grechd G, Franch-Expósitoe S, Briffacd R, et al. The landscape of genomic copy number alterations in colorectal cancer and their consequences on gene expression levels and disease outcome. Molecular Aspects of Medicine. 2019;69, (October 2019):48-61.

27. Pavelka N, Rancati G, Zhu J, Bradford WD, Saraf A, Florens L, et al. Aneuploidy confers quantitative proteome changes and phenotypic variation in budding yeast. Nature. 2010;468(7321):321-5.

28. Sheltzer JM, Torres EM, Dunham MJ, Amon A. Transcriptional consequences of aneuploidy. Proc Natl Acad Sci U S A. 2012;109(31):12644-9.

29. Torres EM, Sokolsky T, Tucker CM, Chan LY, Boselli M, Dunham MJ, et al. Effects of aneuploidy on cellular physiology and cell division in haploid yeast. Science. 2007;317(5840):916-24.

30. Piatkowska EM, Naseeb S, Knight D, Delneri D. Chimeric protein complexes in hybrid species generate novel phenotypes. PLoS Genet. 2013;9(10):e1003836.

31. Olesen K, Felding T, Gjermansen C, Hansen J. The dynamics of the Saccharomyces carlsbergensis brewing yeast transcriptome during a production-scale lager beer fermentation. FEMS Yeast Res. 2002;2(4):563-73.

32. James TC, Campbell S, Donnelly D, Bond U. Transcription profile of brewery yeast under fermentation conditions. Journal of Applied Microbiology. 2003;94:432-48. 
664 33. James TC, Campbell SG, Bond UM. Comparative analysis of global gene expression

665

666

667

668

669

670

671

672

673

674

675

676

677

678

679

680

681

682

683

684

685

686

687

688

689

690

691

692

693

694

695

696

697

698

699

700

701

702

703

704

705

706

707

708

709

710

711

712

in lager and laboratory yeast strains grown in wort. Proceedings of the IEEE.

2002;90:1887-99.

34. Smart KA. Brewing yeast genomes and genome-wide expression and proteome profiling during fermentation. Yeast. 2007;24(11):993-1013.

35. Horinouchi T, Yoshikawa K, Kawaide R, Furusawa C, Nakao Y, Hirasawa T, et al. Genome-wide expression analysis of Saccharomyces pastorianus orthologous genes using oligonucleotide microarrays. J Biosci Bioeng. 2010;110(5):602-7.

36. Somani A, Box WG, Smart KA, Powell CD. Physiological and transcriptomic response of Saccharomyces pastorianus to cold storage. FEMS Yeast Res. 2019;19(4).

37. $\mathrm{Xu} \mathrm{W,} \mathrm{Wang} \mathrm{J,} \mathrm{Li} \mathrm{Q.} \mathrm{Comparative} \mathrm{proteome} \mathrm{and} \mathrm{transcriptome} \mathrm{analysis} \mathrm{of} \mathrm{lager}$ brewer's yeast in the autolysis process. FEMS Yeast Res. 2014;14(8):1273-85.

38. Timouma S, Balarezo-Cisneros LN, Pinto J, De La Cerda R, Bond U, Schwartz JM, et al. Transcriptional profile of the industrial hybrid Saccharomyces pastorianus reveals temperature-dependent allele expression bias and preferential orthologous protein assemblies. Mol Biol Evol. 2021.

39. Timouma S, Schwartz JM, Delneri D. HybridMine: A Pipeline for Allele Inheritance and Gene Copy Number Prediction in Hybrid Genomes and Its Application to Industrial Yeasts. Microorganisms. 2020;8(10):1554-69.

40. Magalhaes F, Vidgren V, Ruohonen L, Gibson B. Maltose and maltotriose utilisation by group I strains of the hybrid lager yeast Saccharomyces pastorianus. FEMS Yeast Res. 2016;16(5).

41. Vidgren V, Londesborough J. Characterization of the Saccharomyces bayanus-type AGT1 transporter of lager yeast. Journal of the Institute of Brewing. 2012;118(2):148-51.

42. Vidgren V, Ruohonen L, Londesborough J. Characterization and functional analysis of the MAL and MPH Loci for maltose utilization in some ale and lager yeast strains. Appl Environ Microbiol. 2005;71(12):7846-57.

43. Lin CL, Garcia-Caro RC, Zhang P, Carlin S, Gottlieb A, Petersen MA, et al. Packing a punch: understanding how flavours are produced in lager fermentations. FEMS Yeast Res. 2021;21(5).

44. Holt S, Miks MH, de Carvalho BT, Foulquie-Moreno MR, Thevelein JM. The molecular biology of fruity and floral aromas in beer and other alcoholic beverages. FEMS Microbiol Rev. 2019;43(3):193-222.

45. van den Broek M, Bolat I, Nijkamp JF, Ramos E, Luttik MA, Koopman F, et al. Chromosomal Copy Number Variation in Saccharomyces pastorianus Is Evidence for Extensive Genome Dynamics in Industrial Lager Brewing Strains. Appl Environ Microbiol. 2015;81(18):6253-67.

46. James T, Usher J, Campbell S, Bond U. Lager yeasts possess dynamic genomes that undergo rearrangements and gene amplification in response to stress. Current genetics. 2008;53(3):139-52.

47. Haimovich G, Medina DA, Causse SZ, Garber M, Millan-Zambrano G, Barkai O, et al. Gene expression is circular: factors for mRNA degradation also foster mRNA synthesis. Cell. 2013;153(5):1000-11.

48. Sun M, Schwalb B, Pirkl N, Maier KC, Schenk A, Failmezger H, et al. Global analysis of eukaryotic mRNA degradation reveals Xrn1-dependent buffering of transcript levels. Mol Cell. 2013;52(1):52-62.

49. Osley MA, Hereford LM. Yeast histone genes show dosage compensation. Cell. 1981;24(2):377-84. 
713 50. Torres EM, Springer M, Amon A. No current evidence for widespread dosage

\section{Figure Legends}

746 Fermentations were carried out in $10 \%$ Wort at $13^{\circ} \mathrm{C}$ in $15 \mathrm{~mL}$ cylindrical tubes. Sugar consumption was measured with a Brixometer. CBS1538: grey line,WS34/70, black line.

748 Error bars represent the standard deviations from the mean of triplicate fermentations. B.

749 Volatile profiles of compounds present in wort at the end of fermentations. Fermentations

750 were carried out in $10 \%$ Wort at $13^{\circ} \mathrm{C}$ in $3 \mathrm{~L}$ tall tubes. Error bars represent the standard 751 deviations from the mean of duplicate fermentations.

753 Figure 2. Chromosome copy number of CBS1538 (A) and WS 34/70 (B) genomes.

754 Estimated copy number and chromosome types for S. eubayanus (blue) and S. cerevisiae 755 (red) isolates from de novo sequencing mapped to the combined parental genomes. The start 756 of each chromosome is shown by a vertical dotted line. *, denotes translocations between $S$. 
757 eubayanus chromosomes II/IV, IV/II, VIII/XV, XV/VIII present in both strains. Hybrid 758 chromosomes are evident from the change in copy number within a chromosome. C. 759 estimated types and copy number of S. cerevisiae (red) and S. eubayanus (blue) and hybrid 760 (red/blue) chromosomes in CBS1538 and WS 34/70.

762 Figure 3. A. Ratio of S. cerevisiae (Sc) and S. eubayanus (Se) in differentially up-regulated 763 and down-regulated transcript pools ( $\log _{2}$-fold change $\geq 1$ or $\leq-1$ ) and mean $\log _{2}$-fold 764 changes under the three conditions in CBS1538 and WS34/70. Ratios of differentially 765 expressed Se:Sc genes with significant differences from the expected are shown with an 766 asterisk, $\mathrm{p} \leq 0.001 * *, \mathrm{p} \leq 0.05^{*}$. B. Venn diagrams showing the relationship of differentially up-regulated and down-regulated transcript pools under the three conditions shown.

Figure 4. Gene ontology analysis of differentially expressed genes in Group I and Group II strains. Genes displaying a $\log _{2}$-fold change $\geq 1$ (A) or $\leq-1$ (B) were examined for enriched gene ontologies using Cluego. Graph shows deviations from the expected ratio of Sc to Se alleles; expected ratio is buff, >expected ratio; buff to red gradient, < expected ratio; buff to blue gradient. Blank cells, no enrichment. Group I expected ratio Sc:Se =0.24:0.76, Group II, Sc:Se $=0.55: 0.45$.

Figure 5. Effect of gene copy number on gene expression patterns. The DEG of Sc and Se orthologues were grouped according to the ratio of gene copy number in WS34/70 (A) and (D) CBS1538. Data for Day 2 in wort relative to minimum medium is shown. The number of paired orthologues at each copy number ratio is shown above the graphs. B. Correlation of differential expression of Sc:Se gene orthologues in WS34/70 with gene orthologue copy number ratio. The mean of $\log _{2}$ fold changes in expression between Sc and Se orthologues is plotted against gene orthologue copy number ratio. Error bars represent the standard error of the mean. C. $\log _{2}$-fold gene expression difference in Sc and Se orthologues on chromosome $\mathrm{X}$ in WS34/70. The dotted line marks the recombination site (THD2) on hybrid chromosomes. Sc:Se ratio to left of vertical dotted line is 3:1 and after 1:1.

Figure 6. Comparison of gene expression in Group I and Group II strains. Transcript reads combined transcriptome. Gene expression profiles in the three conditions, growth in minimal medium, in wort on Day 2 and in wort on Day 4 were compared between the two strains. The 
791 data for Day 2 in wort are shown. A. Venn diagram of genes upregulated in the Group I and

792 Group II strains. B. Gene ontologies enriched in the Group I and Group II strains

793 respectively. White squares, no enrichment, enriched ontologies with the shading represents

794 the percentage of associated genes for each ontology group with the minimum set at $25 \%$.

795 C. $\log _{2}$-fold differences in gene expression between Group I and II strains were grouped

796 according to the ratio of Sc:Se gene copy number. The number of genes in each ratio

797 category is shown above the graphs. Data for Day 2 in wort is shown. The error bars

798 represent the standard error of the mean $\log _{2}$-fold change in gene expression between Group

799 I and II strains.

800 
A

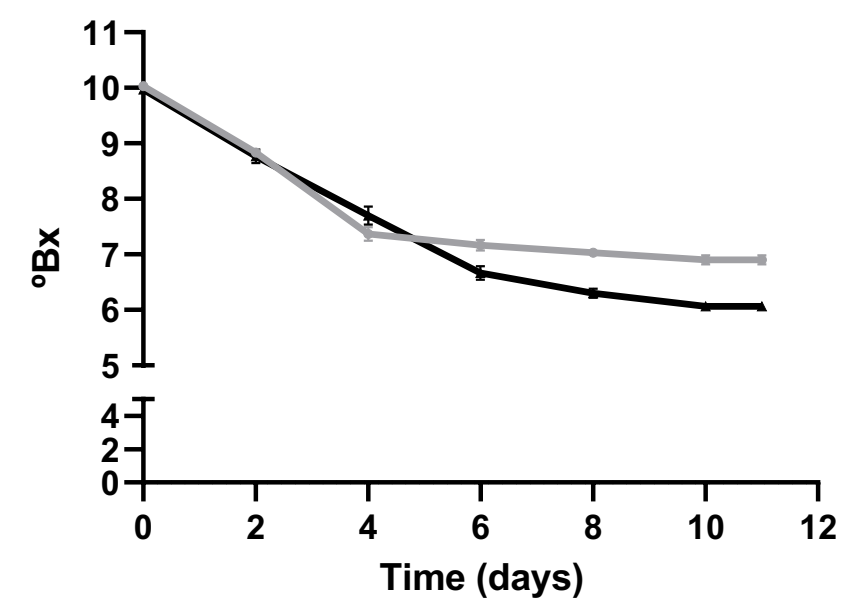

B

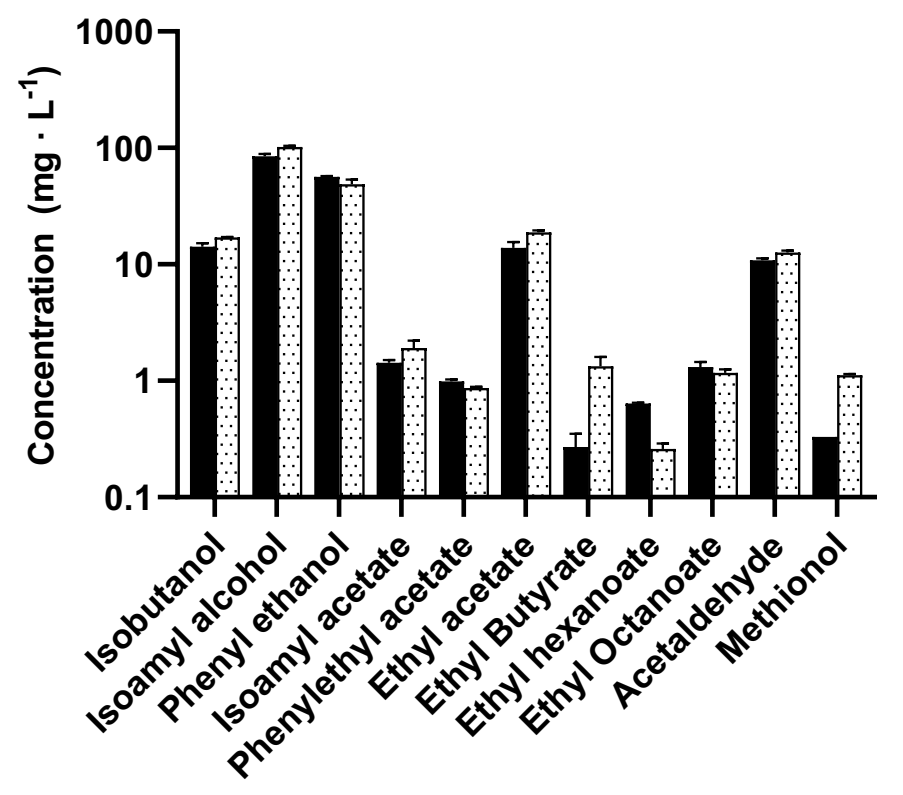

Figure 1 
C

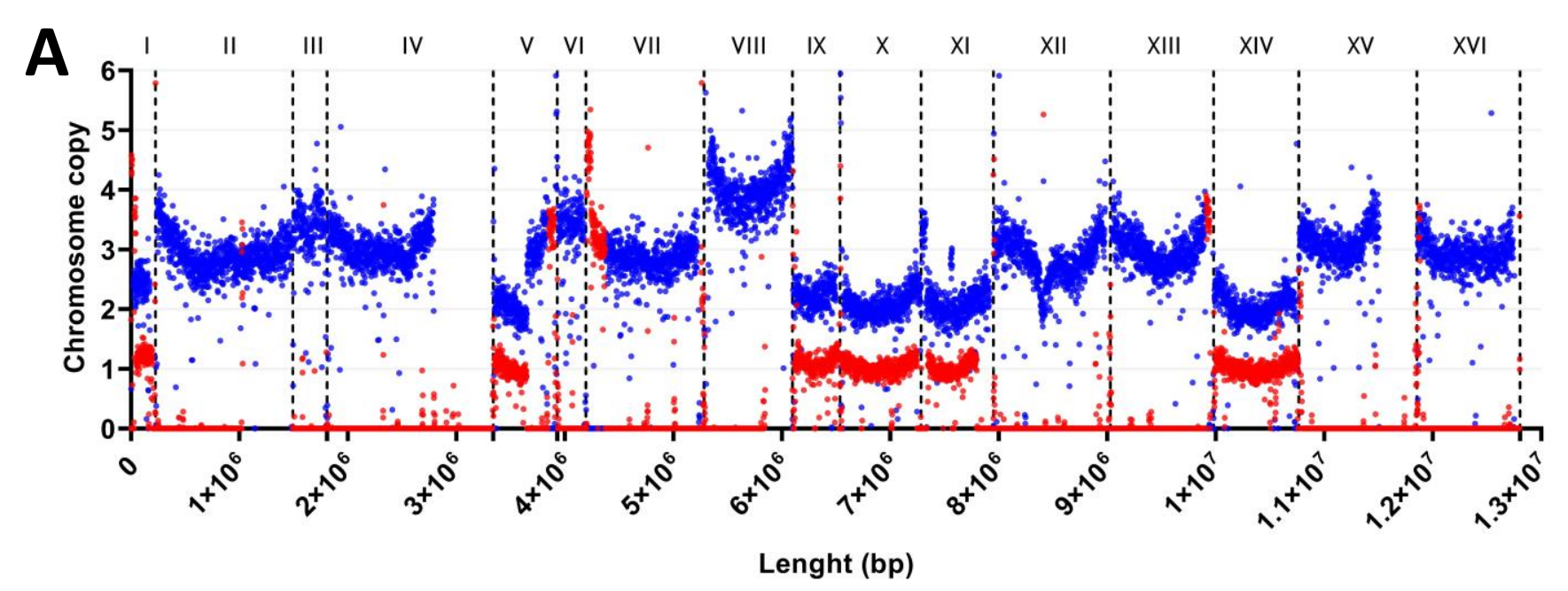

B

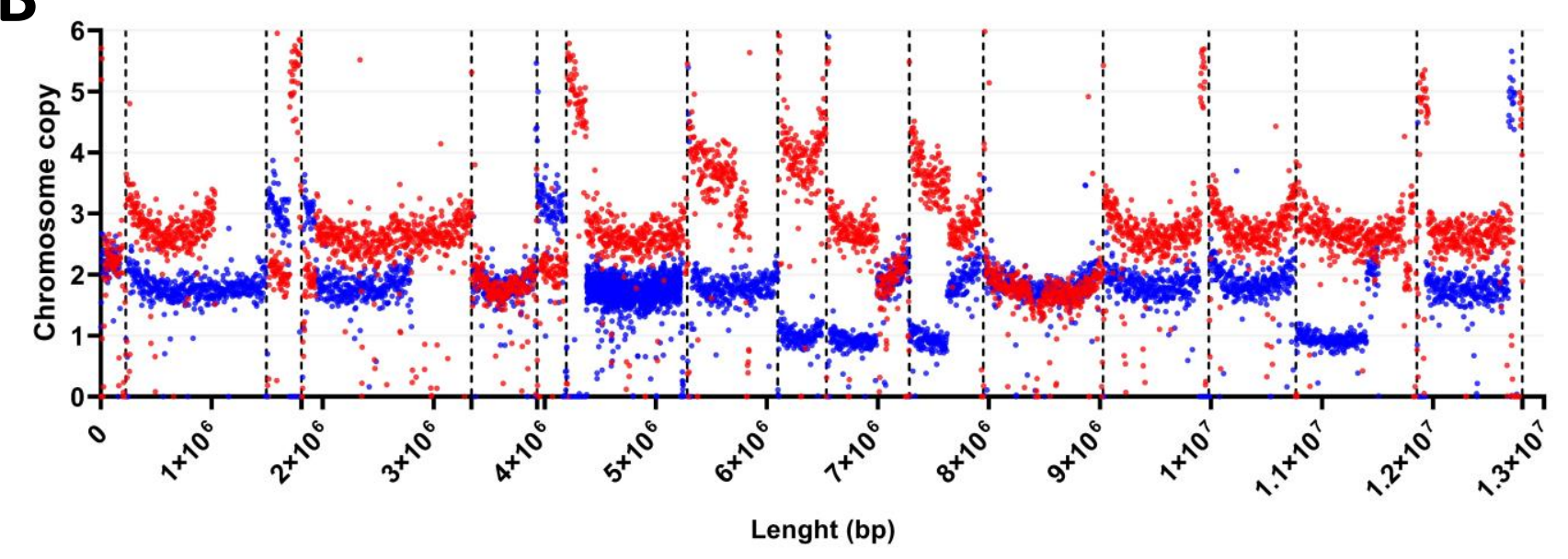

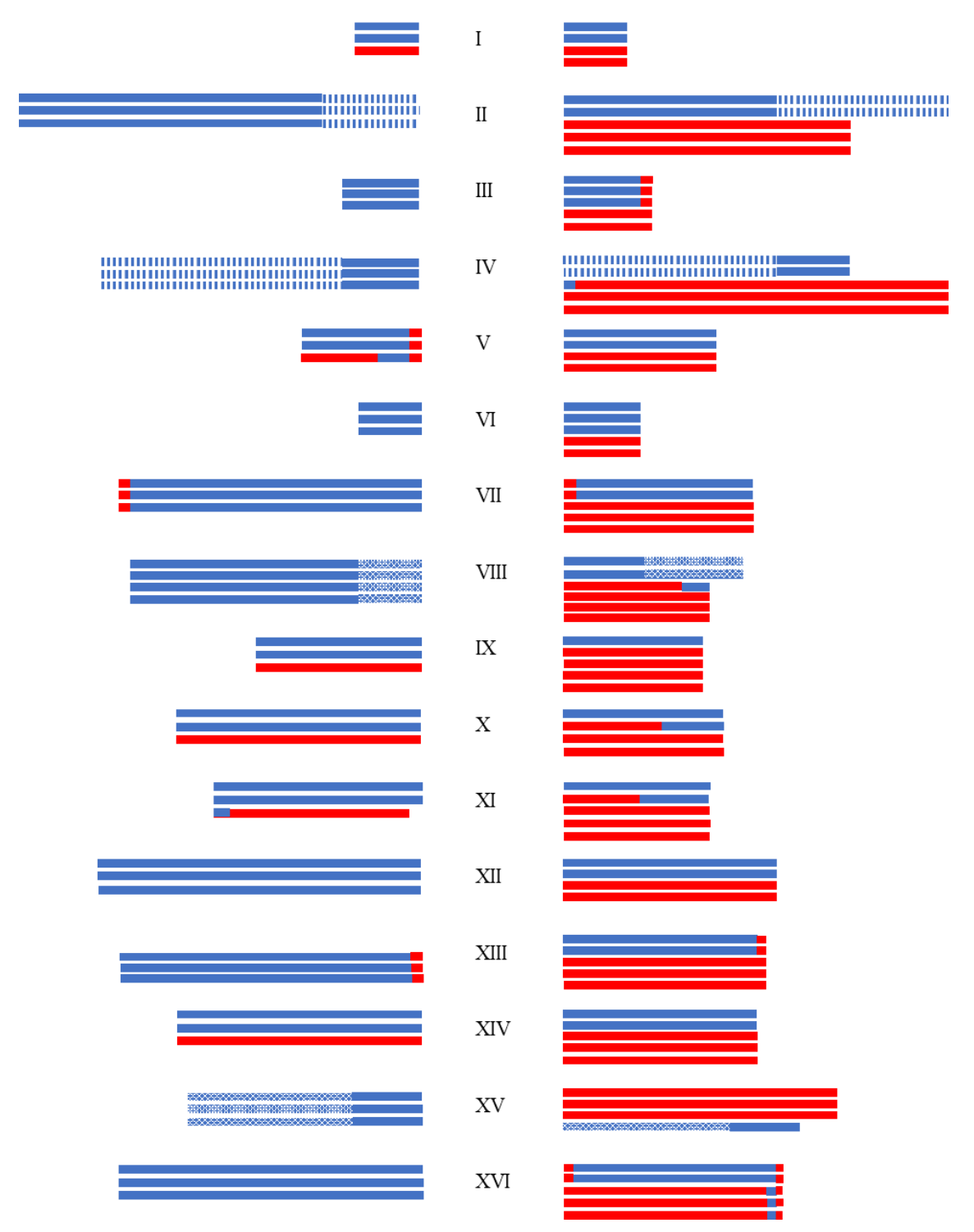

Figure 2 


\begin{tabular}{|c|c|c|c|c|c|c|c|}
\hline & & \multicolumn{2}{|c|}{ D2 vs Min } & \multicolumn{2}{|c|}{ D4 vs Min } & \multicolumn{2}{|c|}{ D2 vs D4 } \\
\hline Strain & & Sc & Se & Sc & Se & Sc & Se \\
\hline \multirow{2}{*}{ WS 34/70 Up } & Ratio Se:Sc & \multicolumn{2}{|c|}{$1.06 * *$} & \multicolumn{2}{|c|}{$0.96^{* *}$} & \multicolumn{2}{|c|}{$1.93 * *$} \\
\hline & $\log _{2}$ fold mean & 2.01 & 2.14 & 2.07 & 2.00 & 1.33 & 1.34 \\
\hline \multirow{2}{*}{ WS 34/70 Down } & Ratio Se:Sc & \multicolumn{2}{|c|}{0.77} & \multicolumn{2}{|c|}{0.77} & \multicolumn{2}{|c|}{0.82} \\
\hline & $\log _{2}$ fold mean & -1.84 & -1.83 & -1.65 & -1.57 & -1.41 & -1.46 \\
\hline \multirow{2}{*}{ CBS 1538 Up } & Ratio Se:Sc & \multicolumn{2}{|c|}{$3.76^{*}$} & \multicolumn{2}{|c|}{2.97} & \multicolumn{2}{|c|}{$4.97^{* *}$} \\
\hline & $\log _{2}$ fold mean & 1.97 & 1.86 & 1.87 & 1.85 & 1.37 & 1.39 \\
\hline \multirow{2}{*}{ CBS 1538 Down } & Ratio Se:Sc & \multicolumn{2}{|c|}{$4.29 * *$} & \multicolumn{2}{|c|}{$3.79^{*}$} & \multicolumn{2}{|c|}{$4.01^{*}$} \\
\hline & $\log _{2}$ fold mean & -1.98 & -1.81 & -1.73 & -1.61 & -1.62 & -1.56 \\
\hline
\end{tabular}
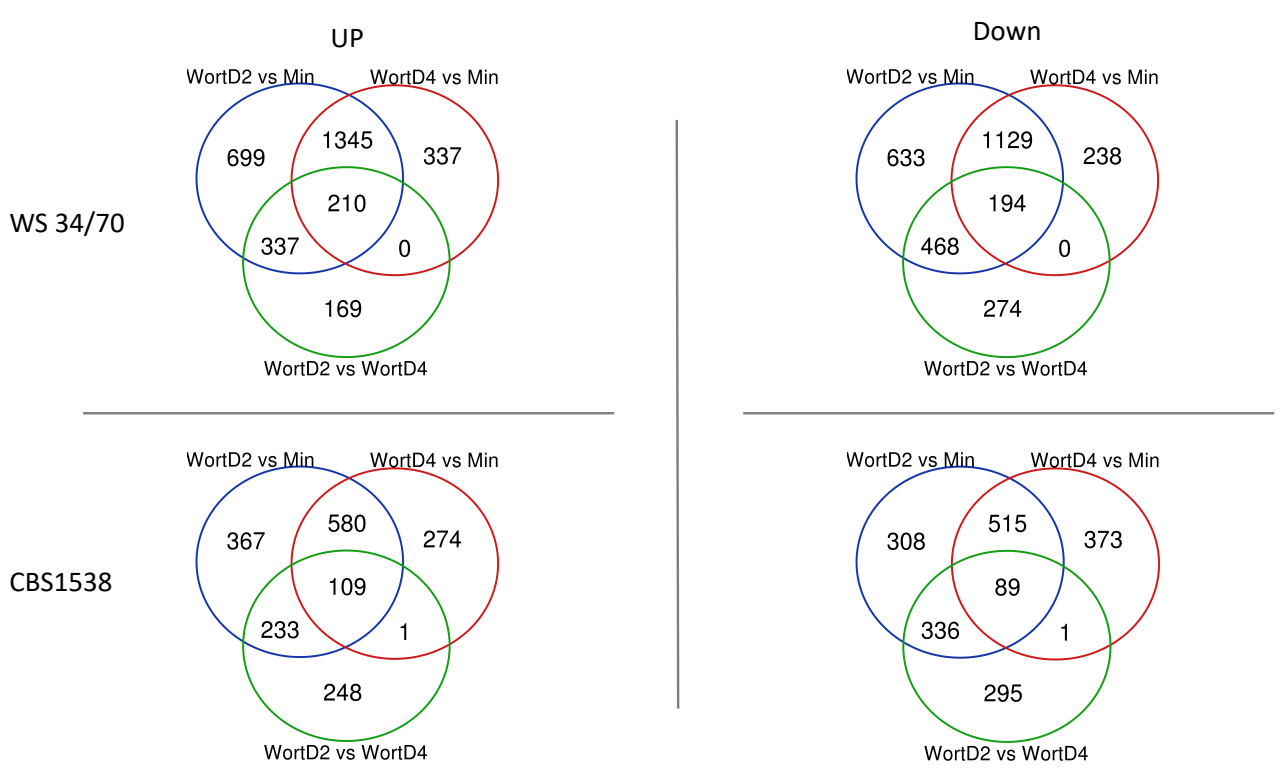

Figure 3 


\section{Grp I Grp II}

Arginine biosynthesis

Alanine, aspartate and glutamate metabolism

Glycine, serine and threonine metabolism

Cysteine and methionine metabolism

Valine, leucine and isoleucine biosynthesis

Lysine biosynthesis Histidine metabolism

Tyrosine metabolism

Phenylalanine metabolism

Phenylalanine, tyrosine and tryptophan biosynthesis

Glutathione metabolism

Pyruvate metabolism

Glycolysis / Gluconeogenesis

Starch and sucrose metabolism -

Fructose and mannose metabolism

Galactose metabolism

Citrate cycle (TCA cycle)

Pentose phosphate pathway

Amino sugar and nucleotide sugar metabolism

Glyoxylate and dicarboxylate metabolism

Glycerolipid metabolism Glycosylphosphatidylinositol (GPI)-anchor biosynthesis

Sphingolipid metabolism

Methane metabolism

Butanoate metabolism

Sulfur metabolism

Ubiquinone and other terpenoid-quinone biosynthesis

Thiamine metabolism

Riboflavin metabolism

Pantothenate and CoA biosynthesis .

Folate biosynthesis

Pyrimidine metabolism

Porphyrin metabolism

$\mathrm{ABC}$ transporters

Autophagy

Protein processing in endoplasmic reticulum

Peroxisome

Longevity regulating pathway

Phagosome
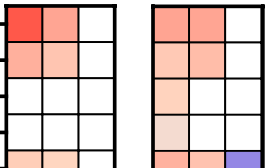

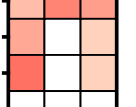
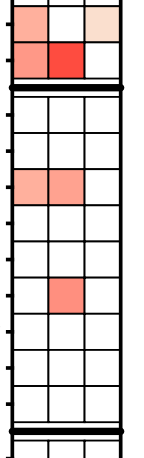

(1)

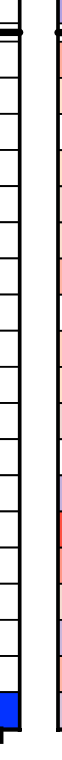
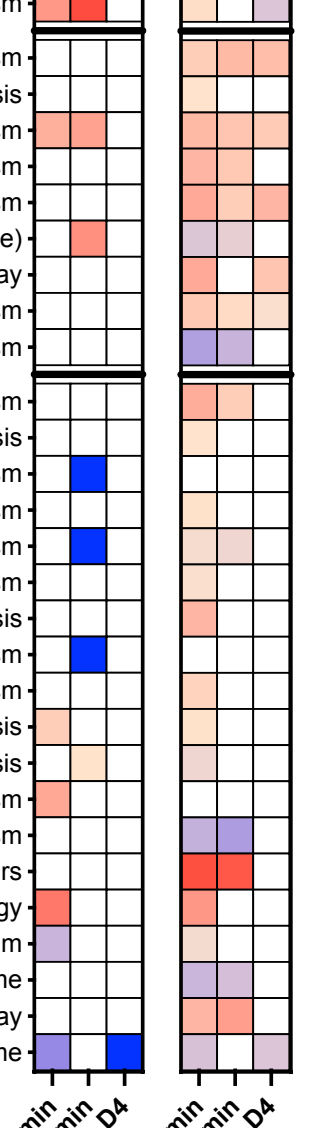

\author{
.
}

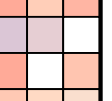

.

\section{.} . . .

\section{Grp I Grp II}

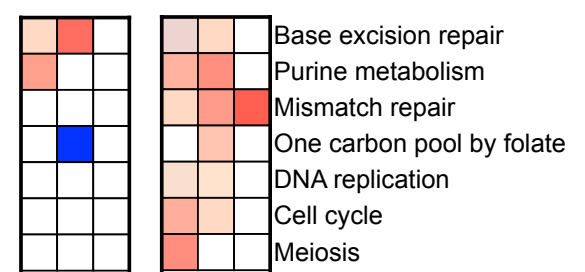

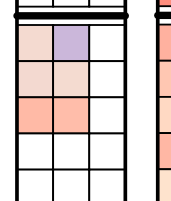

\begin{tabular}{|l|l|l|l}
\hline \hline & & & Ribosome \\
Ribosome biogenesis in eukaryotes \\
RNA polymerase
\end{tabular}

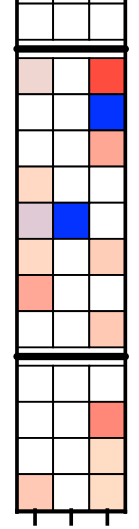

RNA transport

Spliceosome

\section{\begin{tabular}{l|l|l}
\hline \hline & & Biosynthesis of unsaturated fatty acids
\end{tabular} \\ Fatty acid biosynthesis}

Fatty acid degradation

Fatty acid elongation

Steroid biosynthesis

Terpenoid backbone biosynthesis

Glycerophospholipid metabolism

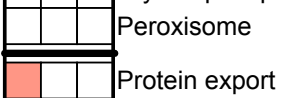

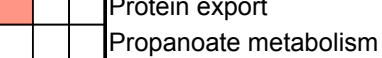

Valine leucine and isoleucine degradation Oxidative phosphorylation

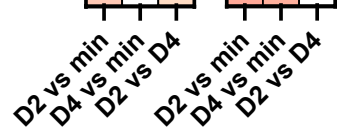

Figure 4 


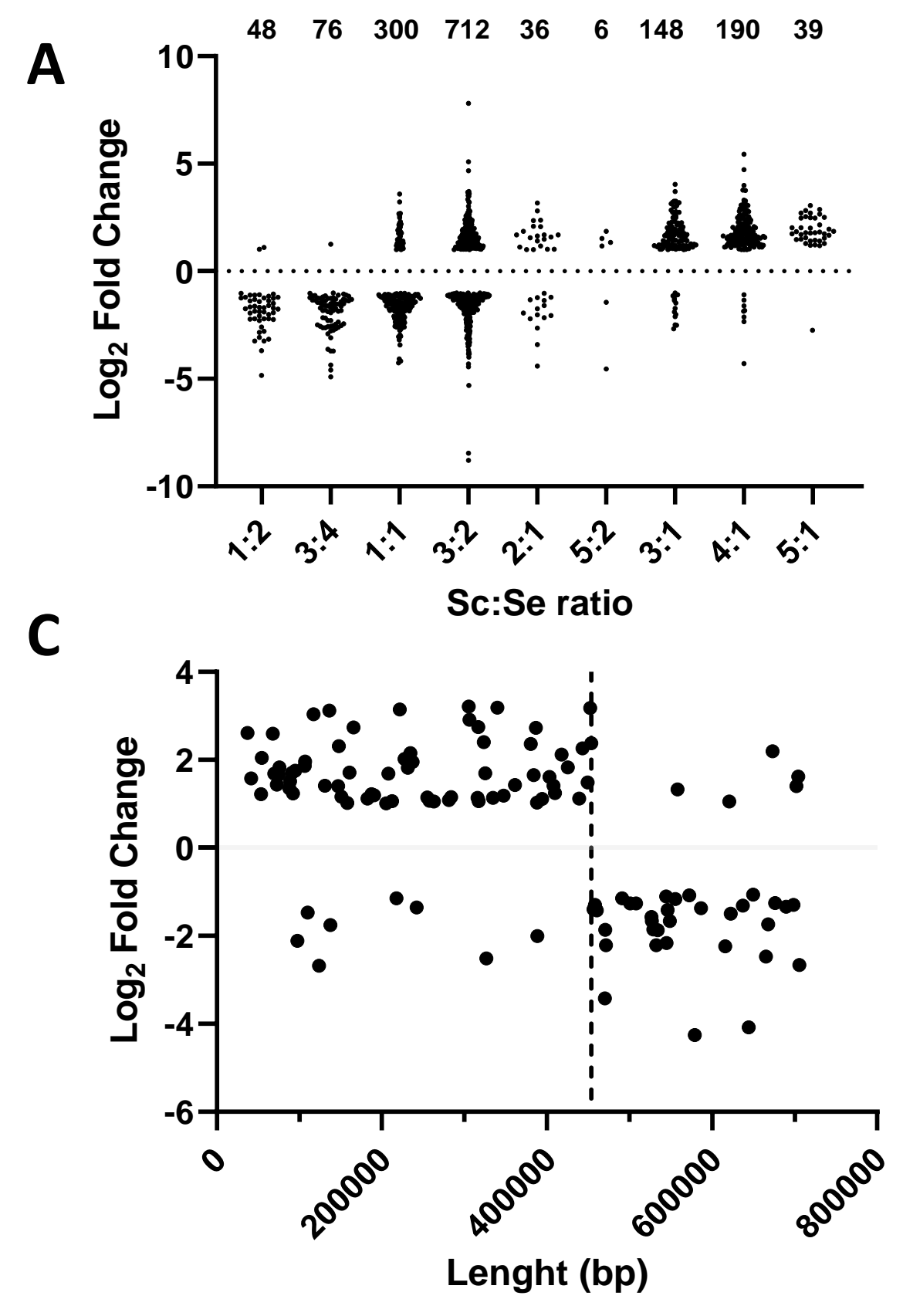

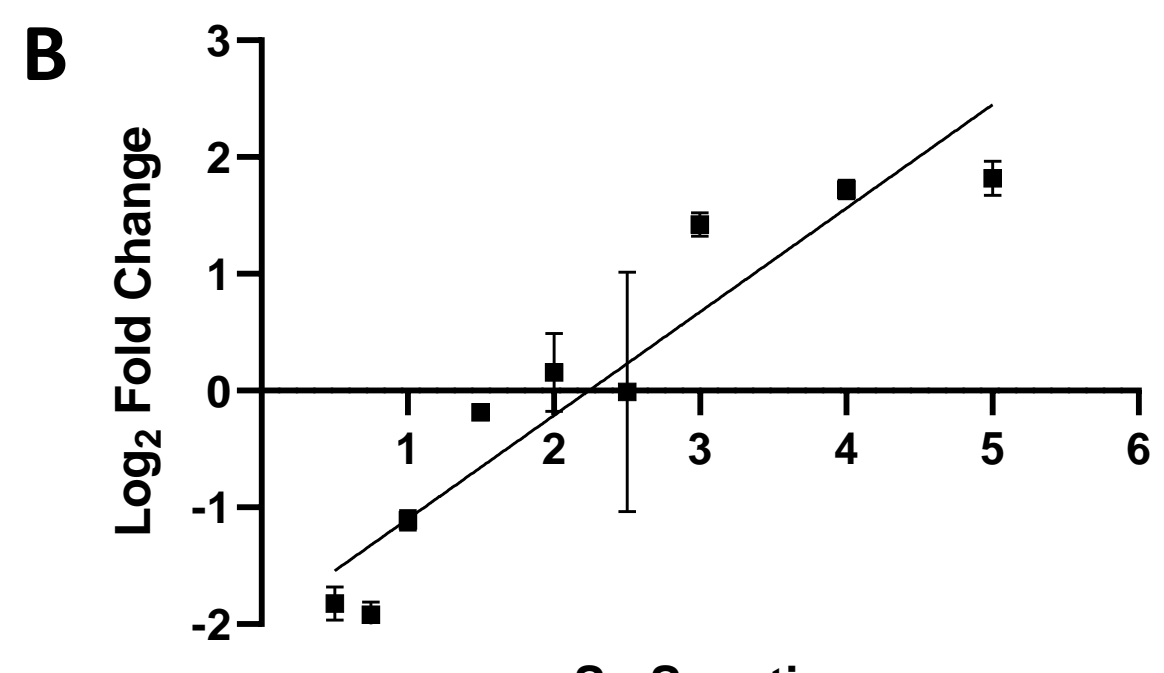

Sc:Se ratio

D

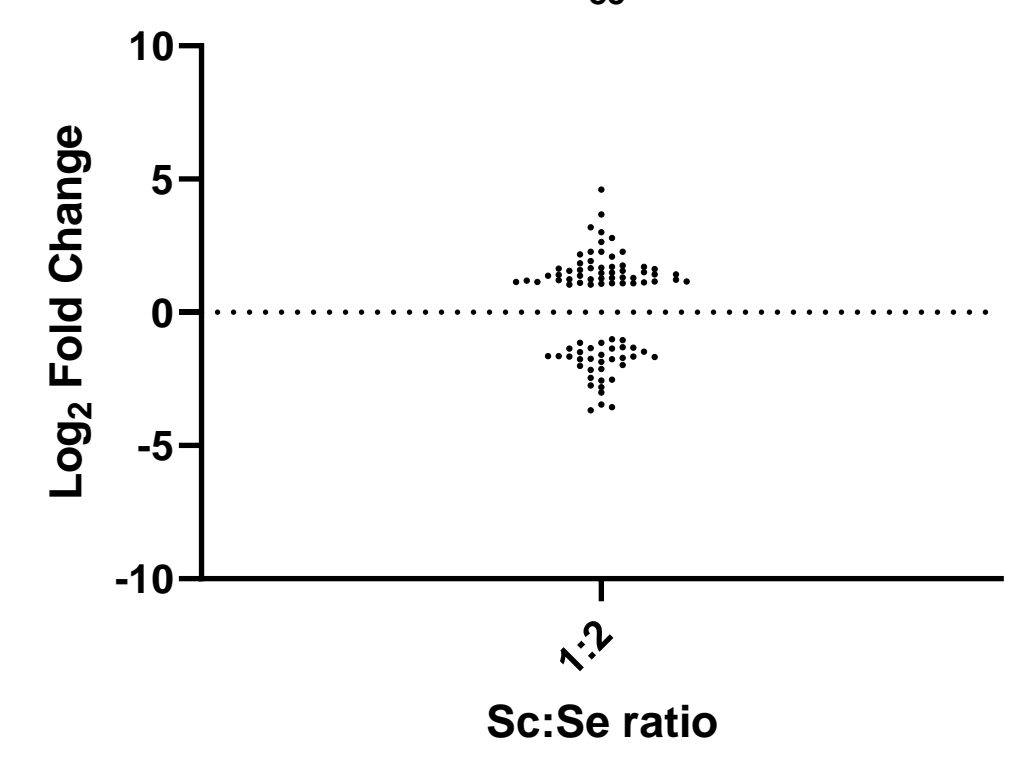

Figure 5 


\section{Group I vs Group II Up}

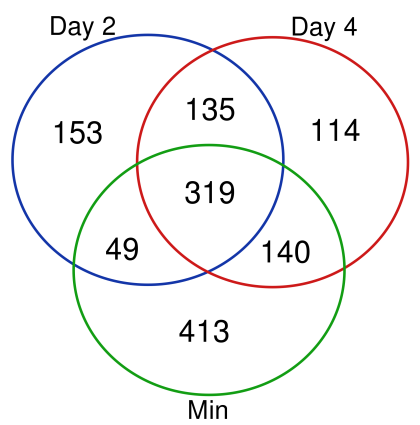

B

Valine, leucine and isoleucine degradation-

Glycolysis / Gluconeogenesis

Galactose metabolism

Fatty acid biosynthesis-

Fatty acid elongation -

Fatty acid degradation-

Steroid biosynthesis -

Tryptophan metabolism-

beta-Alanine metabolism -

Starch and sucrose metabolism -

N-Glycan biosynthesis -

Amino sugar and nucleotide sugar metabolism -

Glycerolipid metabolism -

Sphingolipid metabolism -

Glyoxylate and dicarboxylate metabolism-

Propanoate metabolism -

Terpenoid backbone biosynthesis -

Sulfur metabolism -

Biosynthesis of unsaturated fatty acidsPeroxisome

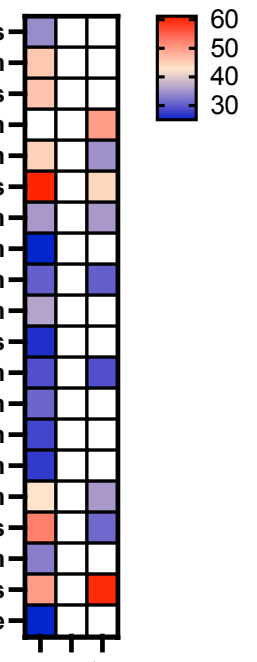

\section{Group II vs Group I Up}
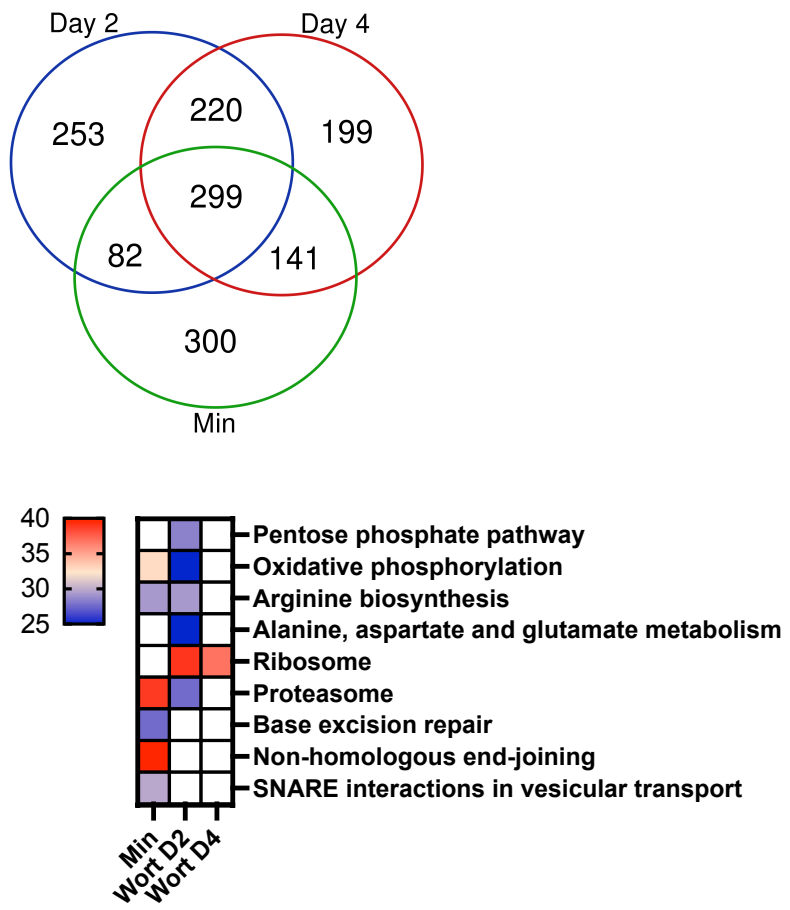

C

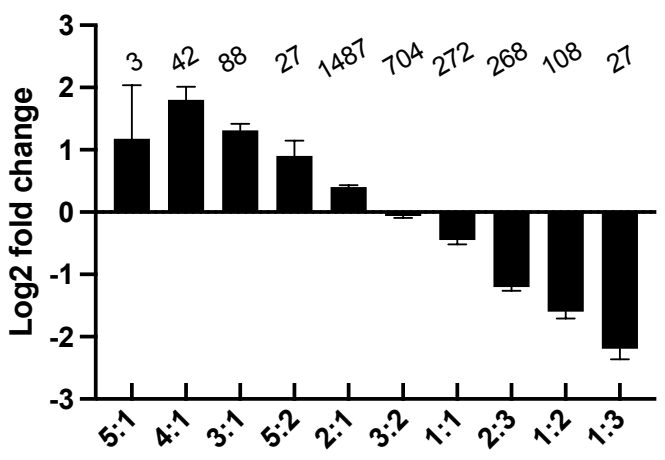

Figure 6 